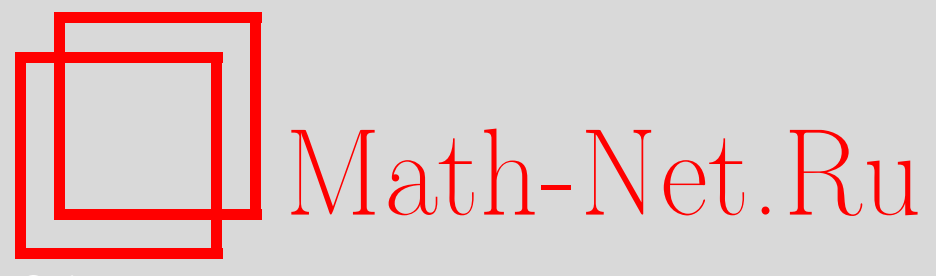

Д. В. Корниенко, Спектральные характеристики нелокальной задачи для двух линейных систем дифференциальных уравнений в частных производных, Вестн. Сам. гос. техн. ун-та. Сер. Физ.мат. науки, 2017, номер 3, 423-436

DOI: https://doi.org/10.14498/vsgtu1558

Использование Общероссийского математического портала MathNet.Ru подразумевает, что вы прочитали и согласны с пользовательским соглашением

http: //www.mathnet.ru/rus/agreement

Параметры загрузки:

IP : 54.147 .182 .235

26 апреля 2023 г., 06:44:50

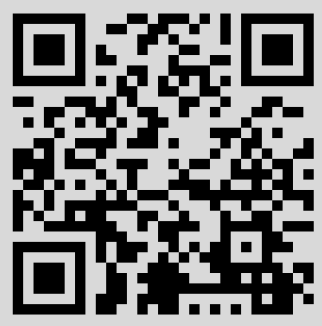


Вестн. Сам. гос. техн. ун-та. Сер. Физ.-мат. науки. 2017. Т. 21, № 3. С. $423-436$ ISSN: 2310-7081 (online), 1991-8615 (print)

УДК 517.956.227

Спектральные характеристики нелокальной задачи для двух линейных систем дифференциальных уравнений в частных производных

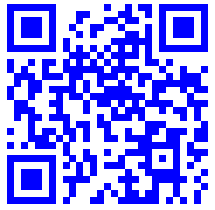

\section{Д. В. Корниенко}

Елецкий государственный университет имени И. А. Бунина, Россия, 399770, Елец, Липецкая обл., ул. Коммунаров, 28.

\section{Аннотация}

Изучается граничная задача для линейной системы дифференциальных уравнений, записанная в виде дифференциально-операторного уравнения

$$
a D_{t} u(t)+b B u(t)=f(t)
$$

с нелокальными граничными условиями по $t$. Такую краевую задачу для линейной системы дифференциальных уравнений (в том числе и в частных производных) мы условимся называть нелокальной.

Цель работы состоит в изучении спектральных характеристик дифференциальных операторов, порожденных нелокальной задачей для двух линейных систем дифференциальных уравнений в частных производных, рассматриваемых в ограниченной области конечномерного евклидова пространства.

Ключевые слова: граничные задачи, нелокальные условия, спектр оператора, эллиптические системы, системы дифференциальных уравнений в частных производных, базис Рисса.

Получение: 15 июля 2017 г. / Исправление: 11 сентября 2017 г. / Принятие: 18 сентября 2017 г. / Публикация онлайн: 28 сентября 2017 г.

\section{Научная статья}

(2) (7) Контент публикуется на условиях лицензии Creative Commons Attribution 4.0 International (https://creativecommons.org/licenses/by/4.0/deed.ru)

\section{Образец для цитирования}

К о р н и ен к о Д. В. Спектральные характеристики нелокальной задачи для двух линейных систем дифференциальных уравнений в частных производных // Вестн. Сам. гос. техн. ун-та. Сер. Физ.-мат. науки, 2017. Т. 21, № 3. С. 423-436. doi: 10.14498/vsgtu1558.

Сведения об авторе

Дмитрий Васильевич Корниенко (D) http://orcid.org/0000-0002-3115-194X

кандидат физико-математических наук, доцент; доцент; каф. прикладной математики и информатики; e-mail: dmkornienko@mail.ru 
Рассмотрим системы уравнений в частных производных вида

$$
\begin{aligned}
& \left\{\begin{array}{c}
D_{t} u^{1}-D_{x} u^{2}-\varepsilon u^{2}=f^{1}, \\
D_{t} u^{2}+D_{x} u^{1}+\varepsilon u_{1}=f^{2} ;
\end{array}\right. \\
& \left\{\begin{array}{r}
-D_{t} u^{1}+D_{x} u^{2}+\varepsilon u^{2}=f^{1}, \\
D_{t} u^{2}+D_{x} u^{1}+\varepsilon u^{1}=f^{2},
\end{array}\right.
\end{aligned}
$$

где $u^{i}=u^{i}(x, t), f^{i}=f^{i}(x, t), i=1,2 ; \varepsilon \in \mathbb{R} ; D_{t}$ - оператор дифференцирования по аргументу $t$; $D_{x}$ - оператор дифференцирования по аргументу $x$.

Системы (1) и (2) будем называть эллиптическими системами первого и второго типа соответственно.

Пусть $t \in V_{t} \equiv[0, T], x \in V_{x} \equiv\left[a_{0}, b_{0}\right] \subset \mathbb{R} ; H_{t}=\mathcal{L}_{2}\left(V_{t}\right), H_{x}=\mathcal{L}_{2}\left(V_{x}\right)$, $H=H_{t} \otimes H_{x}^{2}-$ гильбертовы пространства.

В гильбертовом пространстве $H$ вектор-функций $u=u^{1} e_{1}+u^{2} e_{2}$ аргументов $t$ и $x$ системам (1) и (2) поставим в соответствие следующие дифференциально-операторные уравнения:

$$
\begin{array}{lll}
a D_{t} u(t)+b B u(t)=f(t) & \text { при } & a=\left(\begin{array}{cc}
1 & 0 \\
0 & 1
\end{array}\right), \quad b=\left(\begin{array}{cc}
0 & -1 \\
1 & 0
\end{array}\right), \\
a D_{t} u(t)+b B u(t)=f(t) & \text { при } & a=\left(\begin{array}{cc}
-1 & 0 \\
0 & 1
\end{array}\right), \quad b=\left(\begin{array}{ll}
0 & 1 \\
1 & 0
\end{array}\right) .
\end{array}
$$

Здесь и далее аргумент $x$ для удобства записи опущен. $B: H_{x} \rightarrow H_{x}$-линейный замкнутый неограниченный оператор с плотной в $H_{x}$ областью определения $\mathfrak{D}(B)$, не зависящей от $t \in V_{t}$, для которого все элементы базиса Рисса $\left\{\varphi^{s}\right\}, s \in S$, пространства $H_{x}$ являются собственным элементами оператоpa $B$ :

$$
B \varphi^{s}=B(s) \varphi^{s} \quad \text { при любом } s \in S
$$

(собственному значению $B(s)$ соответствует собственный элемент $\varphi^{s}$ ). Здесь и далее $S$ - некоторое счетное множество индексов $s$, которыми нумеруются элементы $\varphi^{s}$ базиса пространства $H_{x}$. Оператор, для которого существует полная система собственных элементов, образующая базис Рисса в $H_{x}$, принято называть $M$-оператором $[1,2]$. В нашем случае оператор $B=B\left(x, D_{x}\right)-$ обыкновенный дифференциальный оператор на $V_{x}$, порождаемый сильно регулярными краевыми условиями [3]. Будем предполагать, что оператор $B\left(x, D_{x}\right)$ не имеет присоединенных функций.

Положим $B(S)=\{B(s): s \in S\}$ и будем считать, что точечный спектр оператора $B: H_{x} \rightarrow H_{x}$ представим в виде $P \sigma B=B(S)$.

В дальнейшем системы (3) и (4) будем называть квазиэллиптическими (КӨ) системами первого и второго типа соответственно.

K операторным уравнениям (3), (4) присоединим нелокальные краевые условия по $t$ вида

$$
\Gamma_{t} u \equiv \mu u(0)-u(T)=0, \quad \mu \neq 0 \in \mathbb{C} .
$$

Пусть оператор $L\left(D_{t}, B\right) \equiv a D_{t}+b B$ определен на достаточно гладких вектор-функциях $u: \mathbb{R} \rightarrow H_{x}^{2}, u=u(t), u(t)=\left(u^{1}(t), u^{2}(t)\right)^{\top} \in H_{x}^{2}, u^{i}(t) \in H_{x}$, 
$i=1,2$, принадлежащих для каждого $t \in V_{t}$ области определения $\mathfrak{D}(B)$ оператора $B$. Элемент $u(t) \in H$ будем называть решением задачи (3)-(5), если найдется последовательность таких гладких и удовлетворяющих условиям (5) вектор-функций $u_{n}(t) \in \mathfrak{D}(B)$, что

$$
\lim _{n \rightarrow \infty} u_{n}(t)=u(t), \quad \lim _{n \rightarrow \infty} L\left(D_{t}, B\right) u_{n}(t)=f(t) .
$$

Другими словами, мы имеем дело с задачей (3)-(5), понятие решения которой, как легко заметить, использует стандартную процедуру замыкания (расширения) оператора $L\left(D_{t}, B\right)$ при условиях (5). Оператор $L: H \rightarrow H$, определяемый как замыкание в $H$ оператора $L\left(D_{t}, B\right)$, первоначально заданного на гладких вектор-функциях, удовлетворяющих краевым условиям (5), называют сильным расширением оператора $L\left(D_{t}, B\right)$ при условиях $(5)$. В этом случае решение $u=u(t)$ называют сильным решением задачи (3)-(5).

Исследованию свойств задачи Дирихле для эллиптических $(2 \times 2)$-систем посвящены работы А. В. Бицадзе $[4,5]$; сильно и усиленно эллиптические системы изучали М. И. Вишик [6], А. П. Солдатов [7,8].

В настоящей работе проводится сравнительное изучение спектральных свойств краевых задач (3), (5) и (4), (5). Говоря о спектре оператора, мы будем следовать терминологии, принятой в монографии [1]. Резольвентное множество, спектр, точечный (дискретный) спектр и непрерывный спектр оператора $L$ будем обозначать соответственно через $\rho L, \sigma L, P \sigma L$ и $C \sigma L$.

Обозначим $s$-проекцию $[1,2]$ оператора $L$ через $L_{s}$. Исследуем свойства операторов $L_{s}, s \in S$. Имеет место следующая лемма.

Лемма 1. Если $0 \in P \sigma B$, mо есть $B(s)=0$, то спектр $\sigma L_{s}$ оператора $L_{s}: H_{t}^{2} \rightarrow H_{t}^{2}$ совпадает с его точечным спектром $P \sigma L_{s}$. Точечный спектр оператора $L_{s}$ дается формулой

$$
\lambda_{k}=\frac{1}{T}(\ln |\mu|+i \arg \mu+i 2 \pi k), \quad k \in \mathbb{Z} .
$$

Собственному значению $\lambda_{k}$ оператора $L_{s}$ coответствуют две собственнъе вектор-функиии:

$$
u_{m, k}(t)=v(t) e_{m}^{k}(t), \quad m=1,2,
$$

$2 \partial e$

$$
v(t)=\exp \left(\frac{t}{T}(\ln |\mu|+i \arg \mu)\right), \quad e_{m}^{k}(t)=e^{k}(t) e_{m}, \quad e^{k}(t)=\frac{1}{\sqrt{T}} \exp \frac{i 2 \pi k t}{T} .
$$

Система собственных вектор-функиий

$$
\left\{u_{m, k}(t): m=1,2 ; k \in \mathbb{Z}\right\}
$$

оператора $L_{s}$ в пространстве $H_{t}^{2}$ образует

а) ортонормированный базис, если $\mu=1$;

б) базис Рисса, если $\mu \neq 1$.

До каз а тель ст в о. Пусть $\mu=1$. Из равенства $\left(u_{m, k}, u_{m^{\prime}, k^{\prime}}\right)_{H_{t}^{2}}=\delta_{m}^{m^{\prime}} \delta_{k}^{k^{\prime}}$, где $\delta_{k}^{k^{\prime}}$ - функция Кронекера, следует ортонормированность в $H_{t}^{2}$ системы собственных вектор-функций (6) оператора $L_{s}$. 
Предположим, что множество (6) неполно в $H_{t}^{2}$. Тогда существует векторфункция $f \in H_{t}^{2}, f=f^{1}(t) e_{1}+f^{2}(t) e_{2}, f \neq 0$ в $H_{t}^{2}$, ортогональная всем вектор-функциям (6). Так как $\left\{e^{k}(t): k \in \mathbb{Z}\right\}$ - полная ортонормированная в $H_{t}$ система, из равенства $\left(f, e_{m}^{k}\right)_{H_{t}^{2}}=\left(f^{m}, e^{k}\right)_{H_{t}^{2}}$ следует противоречие: $f=0$ в $H_{t}^{2}$ и, следовательно, полнота в $H_{t}^{2}$ системы (6).

Пусть теперь $\mu \neq 0$. Оператор $\mathcal{T}: H_{t}^{2} \rightarrow H_{t}^{2}$ - оператор умножения на непрерывную функцию $v(t)$ является ограниченным и ограниченно обратимым в $H_{t}^{2}$, то есть $0 \in \rho \mathcal{T}^{-1} \cap \rho \mathcal{T}$.

Из равенств $u_{m, k}(t)=\mathcal{T} e_{m}^{k}(t)$ следует, что система (6) образует базис Рисса в $H_{t}^{2}$, а оператор $L$ является $M$-оператором в $H_{x}^{2}$.

Аналогично доказывается следующая лемма.

Лемма 2. Спектр $\sigma L_{s}$ оператора $L_{s}: H_{t}^{2} \rightarrow H_{t}^{2}$ coвnадает с его точечным спектром Р $\sigma L_{s}$. Точечный спектр оператора $L_{s}$ дается формулой

$$
\lambda_{m, k, s}=\frac{1}{T}(\ln |\mu|+i \arg \mu+i 2 \pi k)+i(-1)^{m} B(s), \quad m=1,2 ; k \in \mathbb{Z} .
$$

Собственному значению $\lambda_{m, k, s}$ соответствует собственная вектор-функиия

$$
u_{m, k}(t)=v(t) e^{k}(t) \frac{e_{1}+i(-1)^{m+1} e_{2}}{\sqrt{2}}
$$

оператора $L_{s}$. Система $\left\{u_{m, k}(t): m=1,2 ; k \in \mathbb{Z}\right\}$ собственных векторфункиий оператора $L_{s}$ образует в пространстве $H_{t}^{2}$

а) ортонормированный базис, если $\mu=1$;

б) базис Рисса, если $\mu \neq 1$.

Теперь мы можем сформулировать свойства нелокальной задачи для КЭ системы первого типа.

Tеорема 1. Cпектр $\sigma L$ оператора $L: H \rightarrow H$ состоит из замыкания на комплексной плоскости точечного спектра $P \sigma L$ оператора L. Множество $C \sigma L=\sigma L \backslash P \sigma L$ образует непрерывный спектр оператора L. Точечный спектр оператора $L$ дается формулой:

$$
\lambda_{m, k, s}=v_{k}+i(-1)^{m} B(s), \quad m=1,2 ; k \in \mathbb{Z} ; s \in S,
$$

zde

$$
v_{k}=\frac{1}{T}(\ln |\mu|+i \arg \mu+i 2 \pi k) .
$$

Собственному значению $\lambda_{m, k, s}$ соответствует собственная вектор-функиия onepamopa $L$ :

$$
\varphi^{s} u_{m, k}(t), \quad \text { əде } \quad u_{m, k}(t)=\exp \left(v_{k} t\right) \frac{e_{1}+i(-1)^{m+1}+e_{2}}{\sqrt{2}} .
$$

Cucmeмa

$$
\left\{\varphi^{s} u_{m, k}(t): m=1,2 ; k \in \mathbb{Z} ; s \in S\right\}
$$

собственных вектор-функиий оператора L образуют базис Рисса в пространстве $H$. 
Доказ ательст в о. В силу леммы 2 система вектор-функций (7) является базисом в $H_{t}^{2}$. Следовательно, система вектор-функций (8) является базисом в $H$. Поэтому достаточно доказать, что базис (8) является базисом Рисса в $H$.

Любая вектор-функция $f \in H$ единственным образом раскладывается в ряд

$$
f=\sum_{s} \sum_{m} \sum_{k} f_{m, k, s} \varphi^{s} u_{m, k}, \text { где } f_{m, k, s} \in \mathbb{C} .
$$

Так как система $\left\{\varphi^{s}: s \in S\right\}$ образует базис Рисса в $H_{x}$, справедливо неравенство

$$
C_{1}^{2} \sum_{s} \sum_{m} \sum_{k}\left\|f_{m, k, s} u_{m, k}\right\|_{H_{t}^{2}}^{2} \leqslant\|f\|_{H}^{2} \leqslant C_{2}^{2} \sum_{s} \sum_{m} \sum_{k}\left\|f_{m, k, s} u_{m, k}\right\|_{H_{t}^{2}}^{2},
$$

в котором константы $0<C_{1} \leqslant C_{2}<+\infty$ не зависят от выбора векторфункции $f \in H$. Осталось заметить, что $\left\|\mathcal{T}^{-1}\right\|^{-1} \leqslant\left\|u_{m, k}\right\|_{H_{t}^{2}} \leqslant\|\mathcal{T}\|$, и воспользоваться определением базиса Рисса.

Исследуем свойства задачи (4), (5).

Аналогично лемме 1 доказывается лемма 3. Отметим различия в структуре спектра сравниваемых задач.

Лемма 3. Если $0 \in P \sigma B$, mo есть $B(s)=0$, mo спектр $\sigma L_{s}$ оператора $L_{s}: H_{t}^{2} \rightarrow H_{t}^{2}$ совпадает с его точечным спектром $P \sigma L_{s}$. Точечный спектр оператора $L_{s}$ дается формулой

$$
\lambda_{m, k}=(-1)^{m} \frac{1}{T}(\ln |\mu|+i \arg \mu+i 2 \pi k), \quad m=1,2 ; k \in \mathbb{Z} .
$$

Собственному значению $\lambda_{m, k}$ оператора $L_{s}$ coответствует собственная вектор-функиия

$$
u_{m, k}(t)=v(t) e_{m}^{k}(t)
$$

¿əe

$$
v(t)=\exp \left(\frac{t}{T}(\ln |\mu|+i \arg \mu)\right), \quad e_{m}^{k}(t)=e^{k}(t) e_{m}, \quad e^{k}(t)=\frac{1}{\sqrt{T}} \exp \frac{i 2 \pi k t}{T} .
$$

Cистема $\left\{u_{m, k}(t): m=1,2 ; k \in \mathbb{Z}\right\}$ собственнъх вектор-функиий оператора $L_{s}$ образует в пространстве $H_{t}^{2}$

а) ортонормированный базис, если $\mu=1$;

б) базис Рисса, если $\mu \neq 1$.

Наиболее существенно отличия соответствующих задач проявляются при внимательном сопоставлении результатов леммы 2 и результатов нижеследующих лемм 4, 5 .

Лемма 4. Пусть $0 \in P \sigma$ B. Спектр $\sigma L_{s}$ оператора $L_{s}: H_{t}^{2} \rightarrow H_{t}^{2}$ совпадает с его точечным спектром $P \sigma L_{s}$. Точечный спектр оператора $L_{s}$ дается бормулой

$$
\lambda_{m, k, s}=(-1)^{m} \sqrt{v_{k}^{2}+B^{2}(s)}, \quad m=1,2 ; k \in \mathbb{Z},
$$


əде

$$
v_{k}=\frac{1}{T}(\ln |\mu|+i \arg \mu+i 2 \pi k)
$$

причем в (9) по определению $\lambda_{m, k, s}=(-1)^{m} B(s)$ при $\mu=1 u k=0$. Собственному значению $\lambda_{m, k, s}$ оператора $L_{s}$ соответствует собственная вектор-функиия

$$
u_{m, k, s}(t)=u_{k}^{1}(t) e_{1}+u_{m, k, s}^{2}(t) e_{2},
$$

əде

$$
u_{k}^{1}(t)=v(t) e^{k}(t), \quad u_{m, k, s}^{2}(t) \frac{\lambda_{m, k, s}+v_{k}}{B(s)} v(t) e^{k}(t),
$$

причем в (10) по определению

$$
u_{m, k, s}^{2}(t)=i(-1)^{(m+1)} v(t) e^{k}(t), \text { npu } B(s)=i(-1)^{m} v(k) u \lambda_{m, k, s}=0,
$$

то есть собственному значению $\lambda_{m, k, s}=0$ соответствует одна собственная вектор-функиия независимо от значения $\mathrm{m}$.

Д о каз а тель ст во. Справедливость формулы (9), описывающей распределение собственных значений оператора $L_{s}$ на комплексной плоскости $C$, и представлений (10) соответствующих им собственных функций проверяется достаточно просто.

Если $\lambda \notin P \sigma L_{s}$, то решение $u_{s}=u_{s}(t)$ уравнения $L_{s} u=\lambda u+f$ для $f=f(t) \in C\left(V_{t}\right)$ дается формулой

$$
R_{\lambda} f(t)=\int_{0}^{T} G_{s}(t, \tau, \lambda) f(\tau) d \tau
$$

где матрица Грина $G_{s}(t, \tau, \lambda)$ принадлежит классу $\mathcal{L}_{2}\left(W_{t}\right), W_{t}=V_{t} \times V_{t}$. Аналогично доказательству теоремы 3 в работе [11] получаем включение $\lambda \in \sigma L_{s}$.

Определим последовательность $\left\{\hat{u}_{k}^{1}(t)\right\}_{k=0}^{\infty}$ по правилу

$$
\hat{u}_{2 k}^{1}(t)=u_{k}^{1}(t), k=0,1,2, \ldots ; \quad \hat{u}_{2 k-1}^{1}(t)=u_{-k}^{1}(t), k=1,2,3, \ldots
$$

Точно так же построим последовательность $\left\{\hat{u}_{m, k, s}^{2}(t)\right\}_{k=0}^{\infty}$. Выясним базисные свойства этих последовательностей.

Лемма 5. Последовательность $\left\{\hat{u}_{k}^{1}(t)\right\}_{k=0}^{\infty}$ образует в пространстве $H_{t}$

а) ортонормированный базис, если $\mu=1$;

б) базис Рисса, если $\mu \neq 1$.

До ка з а тель ст в о. Ортонормированность в $H_{t}$ элементов упорядоченного множества $\left\{\hat{e}^{k}(t)\right\}_{k=0}^{\infty}$ проверяется непосредственно. Полнота этого множества в $H_{t}$ вытекает, например, из [9]. Следовательно, $\left\{\hat{u}_{k}^{1}(t)\right\}_{k=0}^{\infty}$ образует ортонормированный базис в пространстве $H_{t}$, если $\mu=1$.

При $\mu \neq 1$ в обозначениях леммы 1 мы имеем $\hat{u}_{k}^{1}(t)=\mathcal{T} \hat{e}^{k}(t)$. Осталось заметить, что в $H_{t}$ оператор $\mathcal{T}$ является ограниченным и ограниченно обратимым. Доказательство закончено. 
Базис Рисса является безусловным базисом. В дальнейшем считаем, что система $\left\{u_{k}^{* 1}(t): k \in \mathbb{Z}\right\}$ биортогональна системе $\left\{u_{k}^{1}(t): k \in \mathbb{Z}\right\}$ и, следовательно, в силу теоремы Банаха [10] является базисом Рисса гильбертова пространства $H_{t}$.

Лемма 6. Пусть $0 \notin P \sigma B$. При фиксированных значениях $m, s$ последовательность $\left\{\hat{u}_{m, k, s}^{2}(t)\right\}_{k=0}^{\infty}$ образует базис в пространстве $H_{t}$; этот базис не является базисом Рисса гильбертова пространства $H_{t}$ для любого $\mu \neq 0 \in \mathbb{C}$.

Доказательство. Положим

$$
u_{m, k, s}^{* 2}(t)=\frac{\overline{\lambda_{m, k, s}}-\overline{v_{k}}}{\overline{B(s)}} \overline{v^{-1}(t)} e^{k}(t), \quad k \in \mathbb{Z} .
$$

Отметим, что собственному значению $\lambda_{m, k, s}=0$ соответствует одна собственная вектор-функция - либо $u_{1, k, s}(t)$, либо $u_{2, k, s}(t)$. Поэтому, если $\lambda_{m, k, s}=0$, то

$$
u_{m, k, s}^{* 2}(t)=i(-1)^{m+1} \overline{v^{-1}(t)} e^{k}(t),
$$

причем либо $m=1$, либо $m=2$.

Последовательность $\left\{\hat{u}_{m, k, s}^{2}(t)\right\}_{k=0}^{\infty}$, построенная из элементов множества $\left\{u_{m, k, s}^{* 2}(t): k \in \mathbb{Z}\right\}$ по правилу (11), биортогональна последовательности $\left\{\hat{u}_{m, k, s}^{2}(t)\right\}_{k=0}^{\infty}$, то есть $\left(\hat{u}_{m, k, s}^{2}, \hat{u}_{m, k^{\prime}, s}^{* 2}\right)_{H_{t}}=\delta_{k}^{k^{\prime}}$ для любых $k, k^{\prime}=0,1,2, \ldots$

Составим для $u \in H_{t}$ формальный ряд

$$
u \sim \sum_{k=0}^{\infty}\left(u, \hat{u}_{m, k, s}^{* 2}\right)_{H_{t}} \hat{u}_{m, k, s}^{2}
$$

Выпишем частичную сумму

$$
S_{n} u=u_{n}=\sum_{k=0}^{n}\left(u, \hat{u}_{m, k, s}^{* 2}\right)_{H_{t}} \hat{u}_{m, k, s}^{2}
$$

ряда (12) и покажем его сходимость в $H_{t}$ :

$$
\lim _{n \rightarrow \infty}\left\|u_{n}-u\right\|_{H_{t}}=0 \text {. }
$$

Так как множество $\left\{u_{m, k, s}^{2}(t): k \in \mathbb{Z}\right\}$ полно в $H_{t}$, для произвольного числа $\varepsilon>0$ линейную комбинацию

$$
a_{N}=\sum_{k=0}^{N} \hat{c}_{k} \hat{u}_{m, k, s}^{2}
$$

выберем так, чтобы $\left\|u-a_{N}\right\|_{H_{t}}<\varepsilon / 2$. Для любого $n \in \mathbb{N}$ имеем

$$
\left\|S_{n} u\right\|_{H_{t}}^{2} \leqslant\|\mathcal{T}\|^{2} \sum_{k=0}^{n}\left|\left(\mathcal{T}^{-1} u, \hat{e}^{k}\right)_{H_{t}}\right|^{2} \leqslant\|u\|_{H_{t}}^{2},
$$


а при $n>N$ очевидно $S_{n} a_{N}=a_{N}$. Следовательно,

$$
\left\|u-u_{n}\right\|_{H_{t}} \leqslant\left\|u-a_{N}\right\|_{H_{t}}+\left\|a_{N}-S_{n} u\right\|_{H_{t}}<\varepsilon
$$

и коэффициенты ряда (12) определены однозначно.

Если последовательность $\left\{\hat{u}_{m, k, s}^{2}(t)\right\}_{k=0}^{\infty}$ является базисом Рисса, то существуют такие константы $C_{q}, q=1,2$, что $+\infty>C_{2} \geqslant C_{1}>0$ и для любой функции $u(t)=v(t) \hat{e}^{2 k}(t), k=0,1,2, \ldots$, независимо от значения $k$ имеем

$$
C_{1}\left|\left(u, \hat{u}_{m, k, s}^{* 2}\right)_{H_{t}}\right| \leqslant\|u\|_{H_{t}} \leqslant C_{2}\left|\left(u, \hat{u}_{m, k, s}^{* 2}\right)_{H_{t}}\right|
$$

Tak как

$$
\left|\left(u, \hat{u}_{m, k, s}^{* 2}\right)_{H_{t}}\right|=\left|\frac{\lambda_{m, k, s}-v_{k}}{B(s)}\right|, \lim _{k \rightarrow \infty}\left|\frac{\lambda_{2, k, s}-v_{k}}{B(s)}\right|=0, \lim _{k \rightarrow \infty}\left|\frac{\lambda_{1, k, s}-v_{k}}{B(s)}\right|=+\infty
$$

в силу (13) неизбежно $C_{q}=C_{q}(k)$ и, следовательно,

$$
\lim _{k \rightarrow \infty} C_{2}(k)=+\infty, \quad \lim _{k \rightarrow \infty} C_{1}(k)=0 .
$$

Полученное противоречие доказывает требуемое.

Таким образом, при фиксированных значениях $m, s$ выделенные нами множества $\left\{u_{k}^{1}(t): k \in \mathbb{Z}\right\}$ и $\left\{u_{m, k, s}^{2}(t): k \in \mathbb{Z}\right\}$ полны в $H_{t}$. Вместе с тем имеет место следующее утверждение.

Лемма 7. Пусть $0 \notin Р \sigma$ В. Тогда справедливы следующие утверждения.

1. При фиксированных значениях $m, s$ множество $\left\{u_{m, k, s}(t): k \in \mathbb{Z}\right\}$ собственных вектор-функиий оператора $L_{s}: H_{t}^{2} \rightarrow H_{t}^{2}$ неполно в $H_{t}^{2}$ для любого $\mu \neq 0 \in C$.

2. Для любого фиксированного $s$ множество $\left\{u_{m, k, s}(t): m=1,2 ; k \in \mathbb{Z}\right\}$ собственных вектор-функиий оператора $L_{s}: H_{t}^{2} \rightarrow H_{t}^{2}$ полно в $H_{t}^{2}$ тогда и только тогда, когда $0 \notin P \sigma L_{s}$.

Доказ ательство.

1. Вектор-функция

$$
f_{m}(t)=e_{k}(t) \overline{v^{-1}(t)}\left(e_{1}+\frac{\overline{v_{k}}-\overline{\lambda_{m, k, s}}}{\overline{B(s)}} e_{2}\right) \neq 0
$$

в $H_{t}^{2}$ и ортогональна всем элементам множества $\left\{u_{m, k, s}(t): k \in \mathbb{Z}\right\}$ при любом фиксированном значении $s$ и для любого $\mu \neq 0 \in C$. В силу критерия полноты получаем требуемое.

2. Пусть вектор-функция $f(t)=f^{1}(t) e_{1}+f^{2}(t) e_{2}$ ортогональна всем элементам множества $\left\{u_{m, k, s}(t): m=1,2 ; k \in \mathbb{Z}\right\}$ собственных вектор-функций оператора $L_{s}: H_{t}^{2} \rightarrow H_{t}^{2}$. Предположим, что $0 \notin P \sigma L_{s}$ и $f(t) \neq 0$ в $H_{t}^{2}$. Из представлений

$$
f^{1}(t)=\sum_{l=-\infty}^{+\infty} f_{l}^{1} u_{l}^{* 1}(t), \quad f^{2}(t)=\sum_{l=-\infty}^{+\infty} f_{l}^{2} u_{l}^{* 1}(t)
$$


и из условия ортогональности получаем условия согласования

$$
f_{k}^{1}+\frac{\overline{\lambda_{m, k, s}}+\overline{v_{k}}}{\overline{B(s)}} f_{k}^{2}=0, \quad k \in \mathbb{Z}
$$

коэффициентов $f_{k}^{1}, f_{k}^{2}$ разложения скалярных функций $f^{1}(t), f^{2}(t)$ в ряды по биортогональным в $H_{t}$ системам функций $\left\{u_{k}^{1}(t): k \in \mathbb{Z}\right\}$, справедливые при $m=1,2$.

Из (15) в силу (14) получаем $f^{2}(t)=0$ и, следовательно, в силу (15) $f(t)=0$ в $H_{t}^{2}$. Противоречие.

Положив $f(t)=u_{k}^{* 1}(t)$, получим вектор-функцию, ортогональную всем элементам множества $\left\{u_{m, k, s}(t): m=1,2 ; k \in \mathbb{Z}\right\}$.

Таким образом, в силу доказанной леммы 7 из системы (10) собственных вектор-функций оператора $L_{s}: H_{t}^{2} \rightarrow H_{t}^{2}$ может быть выделен базис пространства $H_{t}^{2}$ только в случае, когда $0 \notin P \sigma L_{s}$.

Лемма 8. Пусть $0 \notin Р \sigma$ В. Тогда справедливы следующие утверждения.

1. Система (10) собственных вектор-функиий оператора $L_{s}: H_{t}^{2} \rightarrow H_{t}^{2}$ минимальна в гильбертовом пространстве $H_{t}^{2}$.

2. Если $0 \notin P \sigma L_{s}$, то система (10) собственных вектор-функиий опеpатора $L_{s}: H_{t}^{2} \rightarrow H_{t}^{2}$ образует базис в пространстве $H_{t}^{2}$; этот базис не является базисом Рисса в гилъбертовом пространстве $H_{t}^{2}$.

До каз а m eль ство. Для доказательства первого утверждения леммы положим

$$
\begin{gathered}
u_{m, k, s}^{*}(t)=C_{m, k, s} \overline{v^{-1}(t)} e^{k}(t)\left(e_{1}+\frac{\overline{\lambda_{m, k, s}}+\overline{v_{k}}}{\overline{B(s)}} e_{2}\right), \\
C_{m, k, s}=\left\{\begin{array}{cc}
\frac{(\overline{B(s)})^{2}}{\left(\overline{\lambda_{m, k, s}}+\overline{v_{k}}\right)^{2}+(\overline{B(s)})^{2}}, & B(s) \neq i(-1)^{m} \overline{v_{k}}, \\
1 / 2, & B(s)=i(-1)^{m} \overline{v_{k}},
\end{array}\right.
\end{gathered}
$$

где $m=1,2 ; k \in \mathbb{Z}$. Система $\left\{u_{m, k, s}^{*}(t): m=1,2 ; k \in \mathbb{Z}\right\}$ биортогональна системе $\left\{u_{m, k, s}(t): m=1,2 ; k \in \mathbb{Z}\right\}$, то есть $\left(u_{m, k, s}, u_{m^{\prime}, k^{\prime}, s}^{*}\right)_{H_{t}^{2}}=\delta_{m}^{m^{\prime}} \delta_{k}^{k^{\prime}}$ для любых $m, m^{\prime}=1,2 ; k, k^{\prime}=0, \pm 1, \pm 2, \ldots$ Осталось воспользоваться критерием минимальности $[9,10]$.

Рассмотрим вопрос о базисности. Составим для $u \in H_{t}^{2}$ формальный ряд

$$
u \sim \sum_{m=1}^{2} \sum_{k=-\infty}^{+\infty}\left(u, u_{m, k, s}^{*}\right)_{H_{t}^{2}} u_{m, k, s}
$$

и выпишем частичную сумму

$$
S_{n} u=u_{n}=\sum_{m=1}^{2} \sum_{k=-n}^{n}\left(u, u_{m, k, s}^{*}\right)_{H_{t}^{2}} u_{m, k, s}
$$

ряда (16). Повторяя рассуждения, использованные при доказательстве леммы 6, получаем

$$
\lim _{n \rightarrow \infty}\left\|u_{n}-u\right\|_{H_{t}^{2}}=0
$$


Так как коэффициенты ряда (16) определены однозначно, упорядоченная методом суммирования система (10) является базисом $H_{t}^{2}$.

Предположим, что построенный базис является базисом Рисса. Тогда найдутся такие константы $C_{2}, C_{1}$, что $+\infty>C_{2} \geqslant C_{1}>0$ и для любой векторфункции $u=u(t) \in H_{t}^{2}$ справедливо неравенство

$$
C_{1}^{2} \sum_{m=1}^{2} \sum_{k=-\infty}^{+\infty}\left|\left(u, u_{m, k, s}^{*}\right)_{H_{t}^{2}}\right|^{2} \leqslant\|u\|_{H_{t}^{2}}^{2} \leqslant C_{2}^{2} \sum_{m=1}^{2} \sum_{k=-\infty}^{+\infty}\left|\left(u, u_{m, k, s}^{*}\right)_{H_{t}^{2}}\right|^{2} .
$$

Если положим

$$
u=u_{k}=u_{k}(t)=v(t) e^{k}(t)\left(e_{1}+\frac{\lambda_{2, k, s}+v_{k}}{B(s)} e_{2}\right)
$$

то получим вполне очевидное противоречие неравенству (17):

$$
\lim _{k \rightarrow \infty}\left\|u_{k}\right\|_{H_{t}^{2}}=\infty \text { и } \sum_{m=1}^{2} \sum_{k=-\infty}^{+\infty}\left|\left(u, u_{m, k, s}^{*}\right)_{H_{t}^{2}}\right|^{2}=1 .
$$

Теперь мы можем сформулировать свойства нелокальной задачи для КЭ системы второго типа. Начнем со свойств упорядоченных и неупорядоченных подмножеств, составленных из координат собственных вектор-функций соответствующего ей оператора $L: H \rightarrow H$.

Лемма 9. Система $\left\{u_{k}^{1}(t) \varphi^{s}: k \in \mathbb{Z} ; s \in S\right\}$ является базисом Рисса в гильбертовом пространстве $H_{t x}=H_{t} \otimes H_{x}$ для любого $\mu \neq 0 \in \mathbb{C}$.

Доказательств о. Пусть $\left\{e_{k}: k \in \mathbb{N}\right\}$ и $\left\{e_{s}: s \in S\right\}$-ортонормированные базисы пространств $H_{t}$ и $H_{x}$ соответственно и $A: H_{t} \rightarrow H_{t}$ - линейные ограниченные обратимые операторы, для которых $0 \in \rho A \cap \rho B ; A e_{k}=u_{k}^{1}$, $k \in \mathbb{N} ; B e_{s}=\varphi^{s}, s \in S$. Тогда $\left\{e_{k} \otimes e_{s}: k \in \mathbb{N}, s \in S\right\}$ - ортонормированный базис в $H_{t x}$, оператор $A \otimes B: H_{t x} \rightarrow H_{t x}$ является линейным ограниченным оператором, $(A \otimes B)\left(e_{k} \otimes e_{s}\right)=u_{k}^{1} \otimes \varphi^{s}$, причем $0 \in \rho(A \otimes B)$. Тем самым требуемое доказано.

В силу лемм 6-8 получаем нижеследующие свойства нелокальной задачи для КЭ системы второго типа.

Лемма 10. Для $m=1,2$ и для любого $\mu \in \mathbb{C}$ последовательность

$$
\left\{u_{m, k, s}^{2}(t) \varphi^{s}: k \in \mathbb{Z} ; s \in S\right\}
$$

(при любом упорядочении по индексу s; по индексу $k-c \mathcal{~}$ (11)) является базисом в гильбертовом пространстве $H_{t x}=H_{t} \otimes H_{x}$; этот базис не является базисом Рисса в пространстве $H_{t x}$.

Доказательство. В силу леммы 3 и леммы 6 последовательность (18) - базис в $H_{t x}$. При $\tilde{u}=u \varphi^{s}, u=v(t) \hat{e}^{2 k}$, из неравенств

$$
C_{1}\left|\left(u, \hat{u}_{m, 2 k, s}^{* 2}\right)_{H_{t}}\right| \leqslant\|\tilde{u}\|_{H_{t x}} \leqslant C_{2}\left|\left(u, \hat{u}_{m, 2 k, s}^{* 2}\right)_{H_{t}}\right|
$$

следует, что система (18) заведомо не является базисом Рисса в $H_{t x}$. 
Теперь мы можем сформулировать основной результат о базисных свойствах системы собственных вектор-функций нелокальной задачи для квазиэллиптической системы второго типа. Положим

$$
\mathcal{N}=\left\{i(-1)^{m} \frac{1}{T}(\ln |\mu|+i \arg \mu+i 2 \pi k): m=1,2 ; k \in \mathbb{Z}\right\} \backslash\{0\} .
$$

Теорема 2. Зависимость свойств системы собственных вектор-функций оператора $L: H \rightarrow H$ от параметров задачи (4), (5) следующая.

1. Система собственных вектор-функиий оператора $L$ минимальна в гилъбертовом пространстве $H$.

2. Система собственных вектор-функиий оператора $L$ полна в гильбертовом пространстве $H$ тогда и толъко тогда, когда множество $\mathcal{N} \cap P \sigma B$ nycmo, mo ecms $0 \notin P \sigma L$.

3. Если множество $\mathcal{N} \cap P \sigma B$ пусто, то система собственных векторбункиий оператора $L$ образует базис в гильбертовом пространстве H; этот базис не является базисом Рисса в $H$.

Доказательство.

1. Так как система $\left\{\varphi^{s}: s \in S\right\}$ биортогональна системе $\left\{\psi^{s}: s \in S\right\}$, система $\left\{\varphi^{s} u_{m, k, s}(t), \psi^{s} u_{m, k, s}^{*}(t): m=1,2 ; k \in \mathbb{Z} ; s \in S\right\}$ является биортонормированной системой в $H$ на основании результатов леммы 8 . В силу критерия минимальности получаем требуемое.

2. Если множество $\mathcal{N} \cap P \sigma B$ непусто, то $B(s)=i(-1)^{m} v_{k} \neq 0$ и, следовательно, $\lambda_{m, k, s}=0 \in P \sigma L$ при некоторых $m, k, s$. В этом случае векторфункция $f(t)=u_{k}^{* 1}(t)\left(e_{1}-B(s) v_{k}^{-1} e_{2}\right) \psi^{s}$ ортогональна системе

$$
\left\{\varphi^{s} u_{m, k, s}(t): m=1,2 ; k \in \mathbb{Z} ; s \in S\right\}
$$

собственных вектор-функций оператора $L$, то есть (19) не является полной в $H$.

Если множество $\mathcal{N} \cap P \sigma B$ пусто, то из условия $B(s) \neq 0$ следует $\lambda_{m, k, s} \neq 0$. В этом случае в силу леммы 3 и леммы 7 система собственных вектор-функций оператора $L_{s}$ полна в $H_{t}^{2}$ для любого $s \in S$. Таким образом, используя результаты $[11,12]$, получаем полноту системы (19).

3. Доказательство этого предложения проводится как в лемме 10 на основе работ $[11,12]$ и леммы 8.

Конкурирующие интересы. Конкурирующих интересов не имею.

Авторская ответственность. Я несу полную ответственность за предоставление окончательной версии рукописи в печать. Окончательная версия рукописи мною одобрена.

Финансирование. Исследование выполнялось без финансирования. 


\section{Библиографический список}

1. Дезин А. А. Общие вопросы теории граничных задач. М.: Наука, 1980. 208 с.

2. Дезин А. А. Дифференциально-операторные уравнения. Метод модельных операторов в теории граничных задач/ Тр. МИАН. Т. 229 / ред. В. С. Владимиров, Е. Ф. Мищенко. М.: Наука, 2000. 176 c.

3. Михайлов В. П. О базисах Рисса в $\mathscr{L}_{2}(0,1)$ // Докл. АН СCCP, 1962. Т. 144, № 5. С. 981984.

4. Бицадзе А. В. О единственности решения задачи Дирихле для эллиптических уравнений с частными производными // УМH, 1948. Т. 3, №6(28). С. 211-212.

5. Бицадзе А. В. Краевые задачи для эллиптических уравнений второго порядка. М.: Наука, 1966. 203 с.

6. Вишик М. И. О сильно эллиптических системах дифференциальных уравнений // $M a-$ тем. сб., 1951. Т. 29(71), №3. С. 615-676.

7. Солдатов А. П., Митин С. П. Об одном классе сильно эллиптических систем // Дифферени. уравнения, 1997. Т. 33, № 8. С. 1118-1122.

8. Солдатов А. П. О первой и второй краевых задачах для эллиптических систем на плоскости // Дифферени. уравнения, 2003. Т. 39, № 5. С. 674-686.

9. Kaczmarz S., Steinhaus H. Theorie der Orthogonalreihen / Monografie Matematyczne. vol. 6. New York: Chelsea Publ., 1951. viii +296 pp.

10. Садовничий В. А. Теория операторов. М.: Высш. шк., 1999. 368 с.

11. Корниенко Д. В. Об одной спектральной задаче для двух гиперболических систем уравнений // Дифберени. уравнения, 2006. Т. 42, №1. С. 91-100.

12. Корниенко Д. В. О спектре задачи Дирихле для систем дифференциальнооператорных уравнений // Дифферени. уравнения, 2006. Т. 42, № 8. С. 1063-1071. 


\title{
MSC: 35P05
}

\section{Spectral characteristics of a nonlocal problem for two linear systems of partial differential equations}

\section{V. Kornienko}

I. A. Bunin Elets State University,

28, Kommunarov st., Elets, Lipetskaya obl., 399770, Russian Federation.

\begin{abstract}
We study the boundary-value problem for a linear system of differential equations written in the form of differential-operator equations

$$
a D_{t} u(t)+b B u(t)=f(t)
$$

with nonlocal boundary conditions at $t$. Such a boundary value problem for a linear system of differential equations (including partial derivatives), we shall call nonlocal. The purpose of the article is to study the spectral characteristics of differential operators generated by the nonlocal task for the two linear systems of differential equations considered in a bounded region of finite-dimensional Euclidean space.
\end{abstract}

Keywords: boundary value problem, nonlocal conditions, operator spectrum, elliptic systems, systems of differential equations, Riesz basis.

Received: $15^{\text {th }}$ July, $2017 /$ Revised: $11^{\text {th }}$ September, $2017 /$ Accepted: $18^{\text {th }}$ September, $2017 /$ First online: $28^{\text {th }}$ September, 2017

Competing interests. I have no competing interests.

Author's Responsibilities. I take full responsibility for submitting the final manuscript in print. I approved the final version of the manuscript.

Funding. The research has not had any funding.

\section{Research Article}

๖ (ㅇ) The content is published under the terms of the Creative Commons Attribution 4.0 International License (http://creativecommons.org/licenses/by/4.0/)

Please cite this article in press as:

Kornienko D. V. Spectral characteristics of a nonlocal problem for two linear systems of partial differential equations, Vestn. Samar. Gos. Tekhn. Univ., Ser. Fiz.-Mat. Nauki [J. Samara State Tech. Univ., Ser. Phys. Math. Sci.], 2017, vol. 21, no. 3, pp. 423-436. doi: 10.14498/vsgtu1558 (In Russian).

Author's Details:

Dmitriy V. Kornienko (1D http://orcid.org/0000-0002-3115-194X

Cand. Phys. \& Math. Sci., Associate Professor; Associate Professor; Dept. of Applied Mathematics \& Computer Science; e-mail: dmkornienko@mail.ru 


\section{References}

1. Dezin A. A. Obshchie voprosy teorii granichnykh zadach [General questions of the theory of boundary value problems]. Moscow, Nauka, 1980, 208 pp. (In Russian)

2. A. A. Dezin Differential operator equations. A method of model operators in the theory of boundary value problems, Tr. Mat. Inst. Steklova, vol. 229, ed. V. S. Vladimirov, E. F. Mishchenko. Moscow, Nauka, 2000, 176 pp. (In Russian)

3. Mikhailov V. P. On Riesz bases in $\mathscr{L}_{2}(0,1)$, Dokl. Akad. Nauk SSSR, 1962, vol. 144, no. 5, pp. 981-984 (In Russian).

4. Bitsadze A. V. On the uniqueness of the solution of the Dirichlet problem for elliptic partial differential equations, Uspekhi Mat. Nauk, 1948, vol.3, no. 6(28), pp. 211-212 (In Russian).

5. Bitsadze A. V. Boundary value problems for second order elliptic equations, North-Holland Series in Applied Mathematics and Mechanics, vol. 5. Amsterdam, North-Holland Publ., 1968, 211 pp.

6. Vishik M. I. On strongly elliptic systems of differential equations, Mat. Sb. (N.S.), 1951, vol. 29(71), no. 3, pp. 615-676.

7. Soldatov A. P., Mitin S. P. On a class of strongly elliptic systems, Differ. Equ., 1997, vol. 33, no. 8, pp. 1125-1129.

8. Soldatov A. P. On the first and second boundary value problems for elliptic systems on the plane., Differ. Equ., 2003, vol. 39, no. 5, pp. 712-725. doi: 10.1023/A:1026102322259.

9. Kaczmarz S., Steinhaus H. Theorie der Orthogonalreihen, Monografie Matematyczne, vol. 6. New York, Chelsea Publ., 1951, viii +296 pp.

10. Sadovnichii V. A. Theory of operators, Contemporary Soviet Mathematics. New York, Consultants Bureau, 1991, xi+396 pp.

11. Kornienko D. V. On a spectral problem for two hyperbolic systems of equations, Differ. Equ., 2006, vol.42, no. 1, pp. 101-111. doi: 10.1134/S0012266106010083.

12. Kornienko D. V. On the spectrum of the Dirichlet problem for systems of operatordifferential equations, Differ. Equ., 2006, vol.42, no.8, pp. 1124-1133. doi:10.1134/ S0012266106080076. 